\title{
Synthesis of non-spherical gold nanoparticles
}

\section{Tréguer-Delapierre', J. Majimel', S. Mornet ${ }^{1}$, E. Duguet ${ }^{1}$, S. Ravaine ${ }^{2}$}

${ }^{1}$ ICMCB, CNRS, Université Bordeaux 1, 87 Ave du Dr A. Schweitzer, 33608 Pessac Cedex, France 2 CRPP, CNRS, Université Bordeaux 1, 115 Ave du Dr A. Schweitzer, 33600 Pessac, France

\begin{abstract}
Non-spherical gold nanoparticles such as rods (short, long) $(1,2)$, wires, cubes (3), nanocages (4), (multi-)concentric shells (5), triangular prisms (6-7), as well as other more exotic structures such as hollow tubes, capsules (6), even branched nanocrystals (8-9) have garnered significant research attention in the past few years. They exhibit unique and fine-tuned properties which either strongly differ or are more pronounced from those of symmetric, spherical gold nanoparticles. Their unusual optical and electronic properties, improved mechanical properties and specific surface-enhanced spectroscopies make them ideal structures for emerging applications in photonics, electronics, optical sensing and imaging, biomedical labelling and sensing, catalysis and electronic devices among others $(10,11,12,13,14,15,16,17,18)$. Furthermore, some of these anisotropic nanoparticles enable elucidation of the particle growth mechanism, which in turn makes it possible to predict and systematically manipulate the final nanocrystal morphology $(8,19-$ 20). Finally, these anisotropic gold nanomaterials provide templates for further generation of novel materials $(21,22)$.

This article provides an overview of current research in the area of anisotropic gold nanoparticles. We begin by outlining key properties that they possess; we then describe how to control their morphology. Some of the most innovative synthetic strategies are highlighted together with an emphasis on recent results from our laboratories as well as future perspectives for anisotropic gold nanoparticles as novel materials.
\end{abstract}

\section{Interests of anisotropic gold nanoparticles}

Non-spherical gold nanoparticles are known for their appealing optical properties. One of their most interesting optical features is the presence of multiple absorption bands correlated with their multiple axes $(13,14,15,18)$. Such structures can support both propagating and localized surface plasmons. For instance, gold nanorods possess two different resonance modes (in contrast to the single one developed for spherically symmetric gold particles which is relatively independent of size). These two separate modes are due to electron oscillation across and along the long axis of the nanorod and are termed the transverse and longitudinal modes, respectively. The latter of which is extremely sensitive to the aspect ratio of the rod (resonance shifts by approximately $100 \mathrm{~nm}$ for a change in aspect ratio of 1 unit). Additional weaker bands corresponding to quadrupoles may also be observed.

Nanoshells composed of dielectric cores coated with thin metal layer(s) have similar properties to spherical gold nanoparticles in the sense that they also exhibit a single surface plasmon resonance (SPR) absorption. Nanoshells, however, allow tuning of the SPR across the visible and infrared region over a range of wavelengths spanning hundreds of nanometers, far exceeding the spectral range of spherical particles. Moreover, for branched nanocrystals, nanocubes or nanocages, SPR absorption is shifted to longer wavelengths relative to the ordinary green $520 \mathrm{~nm}$ resonance of spherical particles. For example, triangle-branched nanocrystals exhibit a blue color. The plasmon resonance has three bands, corresponding to the two in-plane or longitudinal surface plasmon absorptions at longer and shorter wavelengths and one out-of-plane or transverse plasmon absorption band. The plasmon bands are very sensitive to the length and sharpness of the tips. Thus, those nanostructures also have the advantage of being tunable in their optical properties. In contrast to nanorods, nanoprisms or nanoshell, however, their plasmon resonances cover a lower spectral range.

In summary, a key feature of the non-spherical nanoparticles is that their optical properties vary dramatically with their physical dimensions. The ease of tuning their optical properties gradually with particle size and shape makes them very interesting when compared to traditional organic dye molecules. In contrast to gold nanospheres, their resonance frequency is tuneable over a wide range from blue to nearinfrared and enables one to set the SPR to a wavelength or spectral region specific to a particular application (Figure 1). For instance, the resonance of anisotropic nanoparticles can be positioned in the 'water window' in the near infrared (800$1300 \mathrm{~nm}$ ), where absorption by biomatter is low. Together with the high degree of biocompatibility of gold, these structures show potential in a wide variety of biological applications (optical labels for biosensing events and biomedical labelling) $(1,10,11,16,17)$. It has also been 


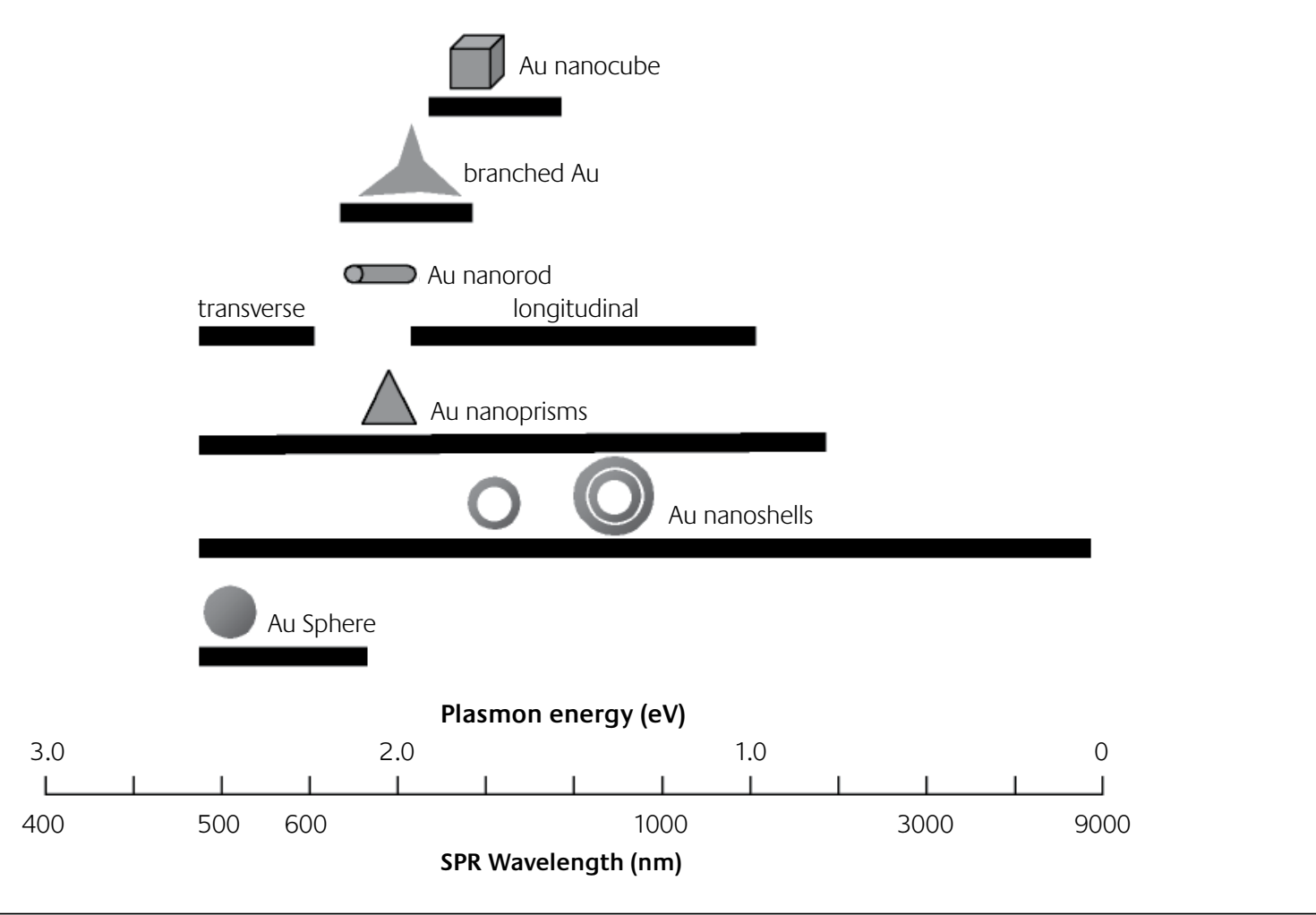

\section{Figure 1}

Range of plasmon resonance of gold nanoparticles as a function of their morphology

speculated that they could play a role in future cancer diagnosis and therapy $(4,5)$. Furthermore, the ability to engineer gold nanoparticles with plasmon resonances in regions of the electromagnetic spectrum appropriate to a particular application also shows potential in fields such as optics, electronics, information technology and in fact all applications which benefit from localized heating $(1,18,23)$. Plasmons indeed play a key role in these different areas.

(Self-)organization of such different nanostructures also opens the door to new methods of studying novel chemical and physical properties arising from collective interactions of the constituting units. It might provide new classes of photonic and electronic devices that control and manipulate light at the nanometer scale, as well as generating, transporting and detecting digital information for homeland security, environmental science, high-speed data communication, and computing components (24).

In addition to photon absorption, heightened surfaceenhanced Raman scattering (SERS) also arises from anisotropic gold nanoparticles. This technique can detect traces of complex molecules, including hazardous agents with molecular specificity. Most experiments were carried out on rough metal films with poorly defined optical properties. However, with gold nanoshells or nanorods for example, the plasmon resonance can be tuned to the excitation of common laser radiation sources optimizing the electromagnetic enhancement mechanism. Moreover, anisotropic particles have highly curved, sharp surface features, with dimensions less than $100 \mathrm{~nm}$ which lead to an increase of the localized electromagnetic field up to one hundredfold at the tip ("lightning rod" effect). The greatest electromagnetic field has been observed at the ends of isolated nanorods or nanowires, compared to spheres, leading to enhanced SERS activity $(25,26,27)$. Another interesting feature of anisotropic nanorods is that their interaction, when assembled, produce huge field enhancement at the nanorod junctions leading to SERS hot spots. Together these features make non-spherical gold nanoparticles promising for all chemical sensing spectroscopies; not only SERS, but also surface-enhanced fluorescence (SEF). The ability to precisely control the interactions between nanoparticles is a powerful tool towards new applications.

Other structures, such as tiny supported anisotropic gold nanoparticles, have been found to have outstanding potential as catalysts. Supported ultra-small gold particles play an important role in a number of industrially relevant reactions such as pollution control, chemical processing, sensors and fuel cell technology $(12,28)$. It is well established that their original catalytic properties are greatly dependent on the particle size $(<5 \mathrm{~nm})$. Shape control over gold nanoparticles may also modulate catalytic activities. Anisotropic nanostructures exhibit different crystal surfaces; they have different fractions of atoms located at different corners, edges and at different defects. Thus, the catalytic efficiency is expected to be different in catalyzing the same reaction. Studies on the catalytic activities of different anisotropic platinum nanoparticles showed pronounced differences for the same electron transfer reaction in colloidal solution (29). For gold, investigations regarding shape dependency on activity and selectivity are still in their infancy (30). This 
(a)

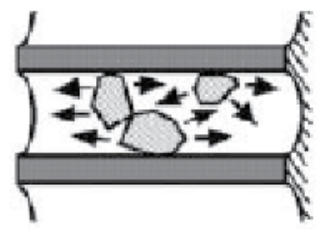

(b)

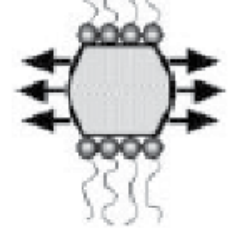

(c)

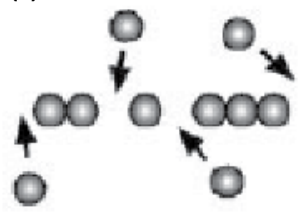

Figure 2

Schematic illustrations of the three strategies developed for controlling the morphology of gold nanoparticles: a) direction through the use of a template; b) kinetic control provided by a capping reagent; c) assembly of preformed spherical nanoparticles

potential shape-dependent catalysis adds to the advantage of using nanoparticles as catalysts. Of course, being small with corners and edges could make their surface atoms unstable during the chemical reaction they catalyze, and shape rearrangements could occur.

\section{Strategies for controlling the shape of gold nanoparticles}

\subsection{Bottom-up routes}

As a colloidal particle is much larger than its constituent atoms, its typical shape mainly results from its surface energy, whose minimization decreases the surface area and favours the formation of spherical objects. In fact, a lot of different preparation routes have been reported for the synthesis of spherical gold colloidal particles (31). However, most of them involving the chemical reduction of $\mathrm{Au}$ ions. For example, reducing agents such as $\mathrm{NaBH}_{4}$ or white phosphorus produce small gold particles (diameter $<10 \mathrm{~nm}$ ) while reductants such as ascorbic acid yield nanoparticles with diameters larger than $10 \mathrm{~nm}$. Since the pioneering work of Turkevich, the most widely studied reductant is sodium citrate (32). It is now well known that, by varying the citrate/Au ratio, colloidal particles with diameters ranging from 10 to $150 \mathrm{~nm}$ may be generated. Noting, however, that for particles of diameter larger than 30 $\mathrm{nm}$ the size distribution widens and the ellipticity of the particles significantly exceeds unity.

In order to control the formation of non-spherical gold nanoparticles, various methodologies have been recently developed (Figure 2). They can be classified as: i) use of templates to shape the particles, ii) use of appropriate capping reagents to kinetically control the growth rates of various facets of a seed, iii) assembly of preformed spherical nanoparticles. Since many of these methods were not demonstrated until recently, most of their characteristics (such as reproducibility, product purity, potential for scalingup, cost effectiveness, and in some cases, mechanism) are only vaguely known. We therefore emphasize here their demonstrated performance (e.g. control of shape) and their intrinsic limits rather than comments on their current level of technological development.

\section{i) Template-directed synthesis}

Template directed synthesis represents a straightforward route to non-spherical gold nanocolloids. In this approach, the template serves as a scaffold within (or around) which gold is generated in situ and shaped into a morphology complementary to that of the template. A wealth of templates have been successfully developed and utilized, including channels within porous materials, mesoscale structures (including Langmuir films) self-assembled from organic surfactants or block copolymers, biological macromolecules such as DNA strains or viruses, and existing nanostructures synthesized using other approaches $(2,31)$. When the template is only physically involved, it is often necessary to remove it selectively using a post-synthesis treatment in order to harvest the resulting gold nanoparticles. It is widely accepted that template-directed synthesis is a simple and cost-effective procedure that also allows a complex topology present on the surface of a template to be duplicated. As a major drawback, the nanoparticles synthesized by this route are often polycrystalline and the quantity that can be produced in each run is relatively limited.

\section{a) Templating against features onto solid substrates}

Relief structures present on the surface of a solid substrate can serve as templates for generating non-spherical gold nanoparticles. In this regard, nanostructures that can be conveniently patterned on the surface of a solid substrate using lithography or photolithography could be exploited as templates. Henzie et al have shown that free-standing gold nanopyramids could be prepared by evaporating gold on an array of pyramidal pits obtained by anisotropically etching the surface of a $\mathrm{Si}(100)$ wafer with a $\mathrm{KOH} /$ isopropyl alcohol solution (33). Penner et al have demonstrated the growth of gold nanowires by templating against the steps on a highly oriented pyrolitic graphite using electrodeposition (34-35). The gold nanowires were found to preferentially nucleate and grow along the step edges present on the graphite surface into a 2D parallel array.

In our laboratory, we have shown the growth of elongated gold nanostructures may occur on specific gold-rich gold sulphide nanoparticle facets (36). Gold salt was reduced by gamma radiation without surfactants. Despite the strong binding energy of gold and sulphur due to the soft character 


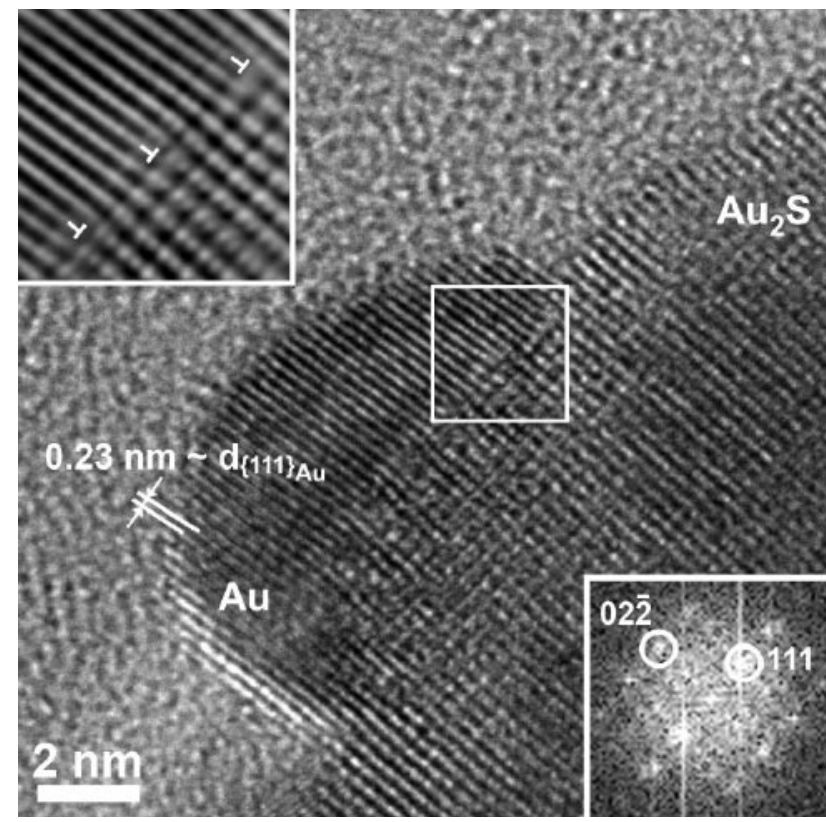

\section{Figure 3}

HRTEM micrograph illustrating the epitaxial growth of gold islands onto a gold sulfide seed (upper inset: interface dislocation network; lower inset: diffraction pattern) (36) (c) 2008 American Chemical Society

of both Au and S, gold preferentially nucleates onto gold rich facets of spherical gold sulphide particles (Figure 3). In order to control the nanocrystal shape and understand the growth mechanism, electronic diffraction and high resolution transmission electronic microscopy (HRTEM) were performed. These techniques have provided us information regarding the nature of the crystal faces and intermediates. We observed that the growth of islands occurs systematically according to the following orientation relationship:

$$
(110)_{A U} / /(110)_{A U_{2} S} ;[1 \overline{1} 1]_{A U} / /[1 \overline{1} 1]_{A U_{2} S}
$$

The (110) plane of the fcc gold sulphide structure is comprised only of gold atoms leading to gold-gold chemical bonds at the interface with the gold nuclei. A dislocation network, perpendicular to the interface plane between the core and the island is formed. The softer material - gold in this system - favours the creation of dislocations to match the lattice misfit (about 18\%) between the two materials rather than elastically deforming.

The addition of a large amount of $\mathrm{Au}^{+}$salt results in the elongation of the gold seeds rather than the formation of new gold nuclei on the initial seed surface. In equilibrium growth conditions, binding energies (Au-Au) are the most important driving force of the directed nucleation and the oriented growth process. In non equilibrium growth conditions, i.e. by very fast reduction rate of $\mathrm{Au}^{+}$salt, the growth of many randomly oriented gold particles is observed. A large number of nuclei merge simultaneously over the surface of the seed particles in the early stage of the reaction, resulting in a layer of larger closely packed nanoparticles (Figure 4). This procedure opens a potential route to the synthesis of gold nanoshells of differing thickness.

By using $\mathrm{CeO}_{2}$ as seeds, gold nanoparticles can also be produced by deposition-precipitation (37). Depending on the $\mathrm{pH}$, temperature, stirring and washing conditions the particle size can be smaller than $5 \mathrm{~nm}$ (Figure 5). Two kinds of orientation relationships have been found between gold and ceria:

$(111)[-110]_{\mathrm{CeO}_{2}} / /(111)[-110]_{\mathrm{Au}} ;(111)[-110]_{\mathrm{CeO}_{2}} / /(111)[1-10]_{\mathrm{Au}}$

Such a crystallographic organization has been previously observed on other noble metal / oxide catalyst materials (38). The lattice mismatch value at the interface is about $25 \%$ whereas the $\mathrm{Au} / \mathrm{CeO}_{2}$ system (just as the $\mathrm{Au} / \mathrm{Au}_{2} \mathrm{~S}$ one) could
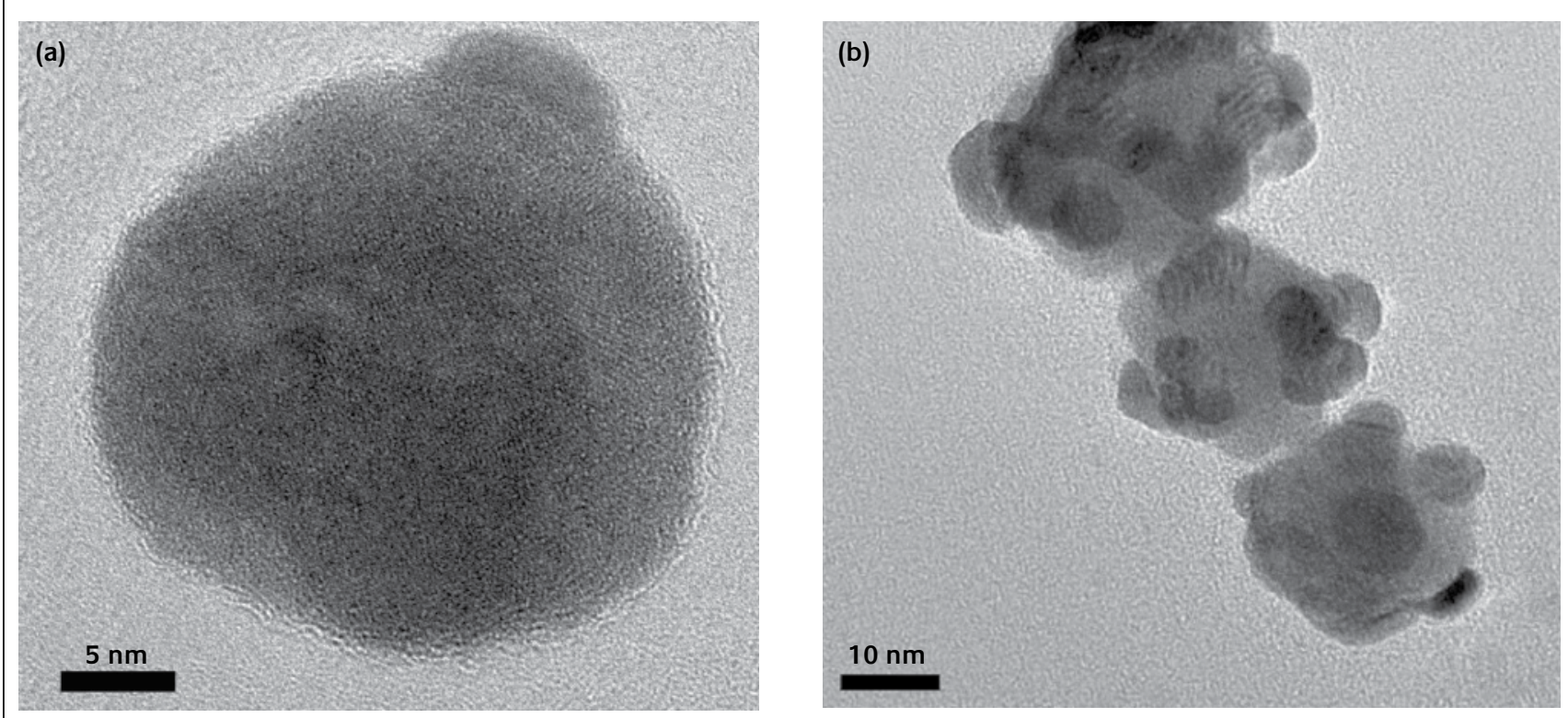

Figure 4

HRTEM micrographs showing typical morphologies of gold deposited onto Au $\mathrm{S}$ colloidal seed particles: a) at low dose rate, b) at high dose rate (36) 
have favoured a lower lattice misfit by adopting another orientation relationship. The crystallographic nature of the interface plane seems to play a key role in such gold-deposited systems.

\section{b) Channels in porous materials}

Channels in porous membranes provide another class of templates for use in the synthesis of non-spherical gold nanostructures (Figure 6). Two types of porous membranes are generally used: polymer films containing track-etched channels and alumina films containing anodically etched pores. This method is derived from the pioneering works of Martin and Moskovits, who have shown the electrochemical deposition of gold nanorods inside porous membranes $(39,40,41)$. They showed that, by changing the aspect ratio of the prepared nanocylinders, the colour of the composite membrane can be varied $(42,43)$. Initially, the template method was employed to prepare microscopic electrodes by depositing gold on a polycarbonate membrane using electrochemical plating methods (44). Subsequently, the method has been applied to the redispersion of the templatesynthesized gold nanorods into water (45). Alternatively, the rods could be dispersed into organic solvents through the dissolution of the appropriate membrane followed by polymer stabilization (46). The diameter of the gold nanoparticles that are synthesized by this way coincides with the pore diameter of the alumina membrane. This means that gold nanorods with different diameters can be prepared by controlling the pore diameter of the template $(39,47)$. The length of the nanorods can be controlled through the amount of gold deposited within the pores of the membrane (48). Similar techniques, using different membranes, have also been successfully applied to the synthesis of gold nanotubes $(49,50,51)$. The fundamental limitation of this template

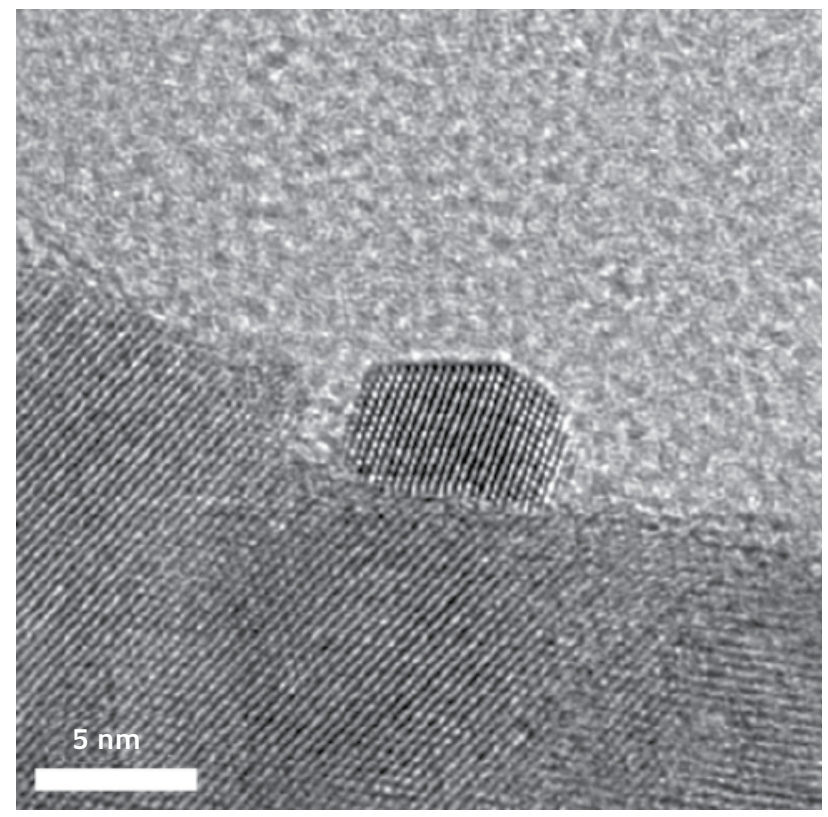

\section{Figure 5}

HRTEM micrograph of a gold island deposited onto a $\mathrm{CeO}_{2}$ nanoparticle (37) method is the yield: since only monolayers of rods are prepared, even milligram amounts of rods are arduous to prepare.

Yan and Goedel have used porous polymer membranes, which have been prepared at the water surface using monolayers of silica colloids, as templates to synthesize gold nanorings (52). The synthesis involves the filling of the pores with a solution of gold precursor followed by calcinations. The size of the rings can be easily controlled by preparing membranes with suitable pore sizes.

In addition to macroporous membranes, mesoporous silica materials have been actively explored in the MCM series and SBA family (53). Preparation of gold nanoparticles in these
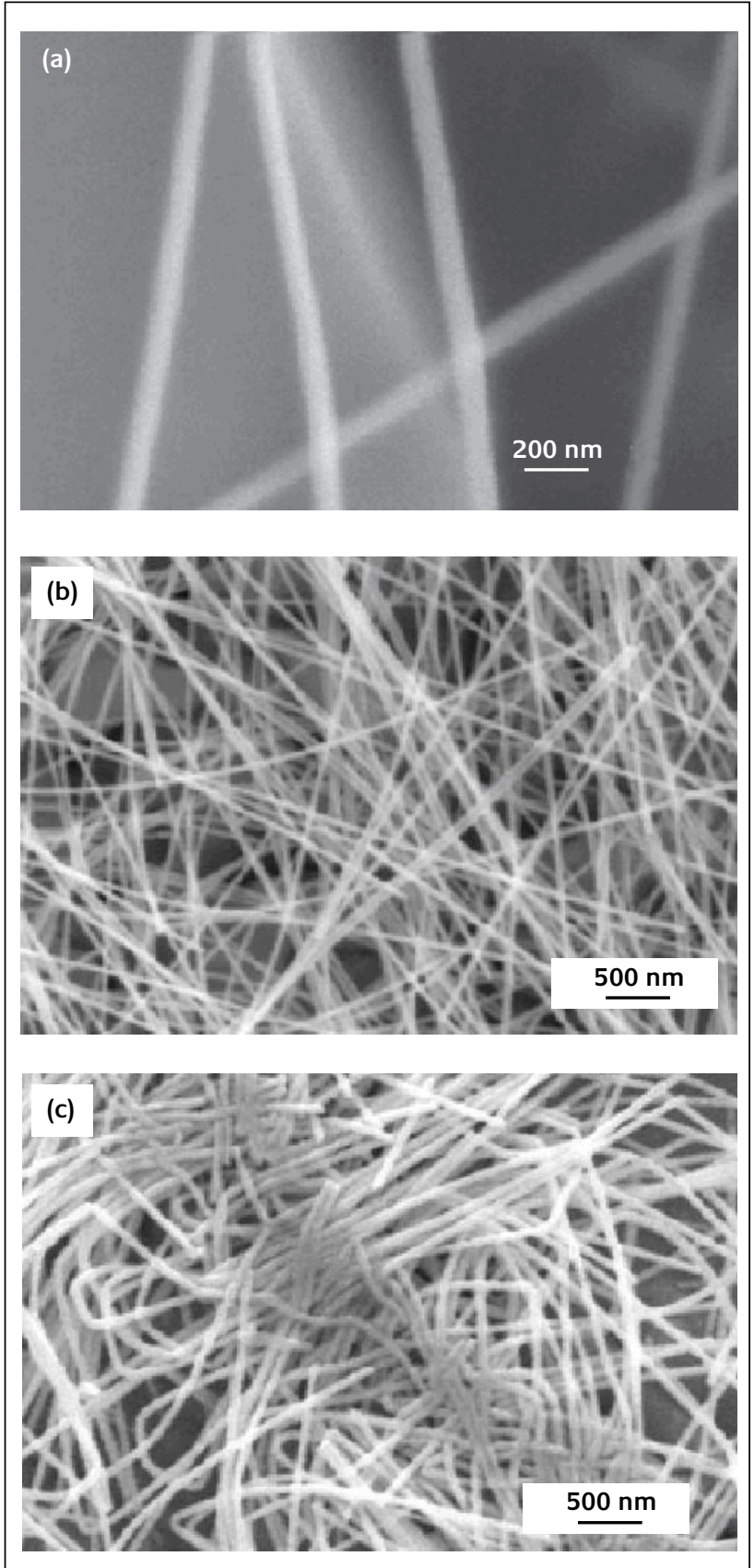

\section{Figure 6}

SEM images of Au nanowires a) single-crystalline, b) and c) polycrystalline synthesized in etched ion-track membranes (119). () 2006 Institute of Physics Publishing 


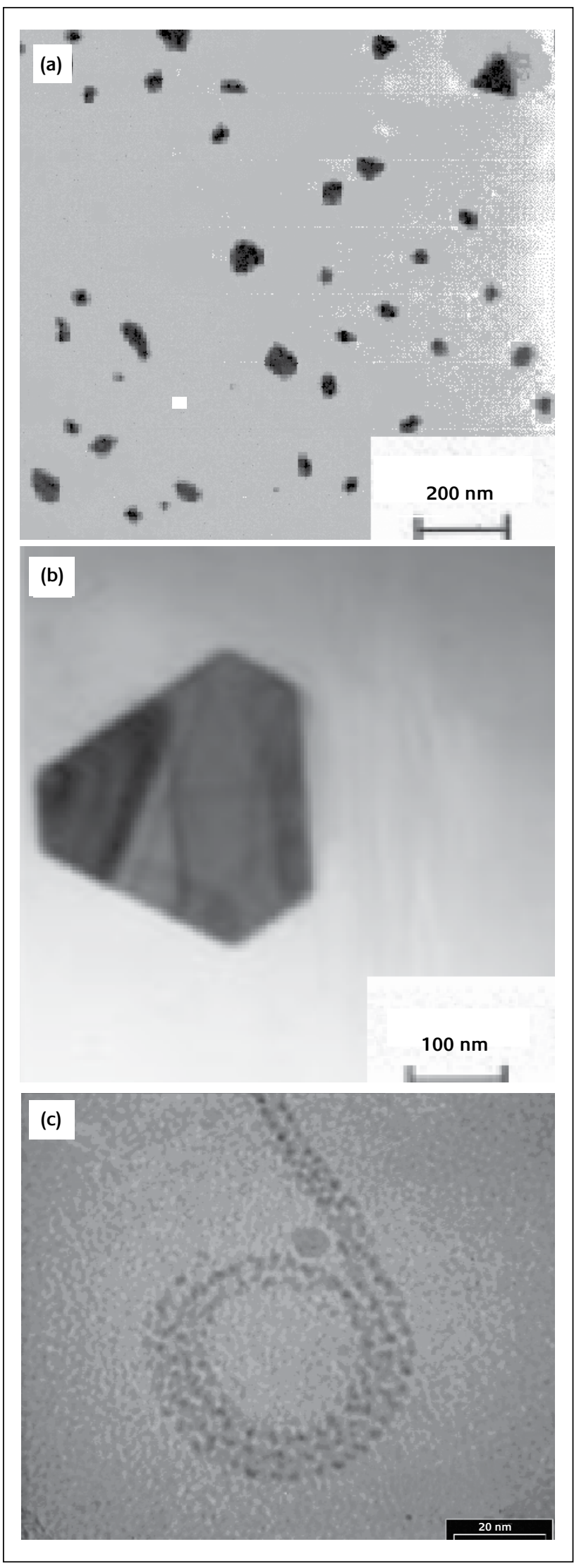

\section{Figure 7}

TEM micrographs of a) gold nanoparticles grown in a nine layer $L B$ film of octadecylamine irradiated for 120 min b) triangular gold single crystals with truncated corners, grown in a nine layer $L B$ film of benzyldimethylstearylammonium chloride monohydrate after exposure to UV irradiation for 120 min c) gold nanoparticles grown in lamellar phases $(59,60)$ templates also involves three steps: infiltration of the pores with an $\mathrm{Au}^{3+}$ salt, reduction of this salt, and recovery of the metallic nanoparticles by selectively removing the template.

c) Templating against self-assembled molecular structures Mesophase structures self-assembled from surfactants provide another class of useful templates for generating nonspherical gold nanoparticles. It is well known that surfactant molecules spontaneously organize into micelles in aqueous media (or reverse micelles in organic solvent systems) when their concentration reaches a critical value. Water is readily solubilized in the polar core of reverse micelles, forming a so-called water pool. These spatially and dimensionally constrained water pools can be used as soft templates to promote the formation of gold nanoparticles with various shapes. Based on this principle, Wang et al demonstrated the synthesis of gold nanorods by templating against rod-like micelles assembled from cethyltrimethylammonium bromide (CTAB) and a small amount of a much more hydrophobic cationic surfactant (tetradodecylammonium bromide $\mathrm{TC}_{12} \mathrm{AB}$ ), which acts as a rod-inducing co-surfactant (54). This method was later exploited by El-Sayed to synthesize gold nanorods with well-controlled aspect ratios (55). Similar nanoparticles have been obtained by Murphy et al by using a seed-mediated growth in the presence of rod-like micelles assembled from CTAB (56). Later, the same group reported an improved methodology to produce monodisperse gold nanorods of high aspect ratio in $~ 90 \%$ yield simply through appropriate $\mathrm{pH}$ control (57).

Block-copolymers (macromolecules formed by connecting two or more chemically distinct segments end-to-end with a covalent bond) can also be exploited as soft templates to generate non-spherical gold nanostructures. When the polymer segments are immiscible and under appropriate conditions, mesophases can be formed. Different regions in such phases can be selectively infiltrated with a gold salt, making block copolymers a powerful system of templates for the synthesis of gold nanoparticles of various shapes (58).

Precipitation under Langmuir films has provided insight into the nature of the templating process not always available to those investigators synthesizing particles in other mesophase systems. The role of the polar head group in binding the precipitating species and relative packing of the amphiphilic molecules can be examined to determine the epitaxial relationship between the template and the templated materials.

We have examined the formation of gold particles using several different amphiphilic molecules using the Langmuir film (59). Ultraviolet radiation was used to induce photoreduction of the gold chloride in the system. It was found that binding between the $\mathrm{AuCl}_{4}^{-}$ions and positively charged polar head groups resulted in templating with the formation of plate-like gold particles from 20 to $800 \mathrm{~nm}$ across the face (Figure $7 \mathrm{a}, \mathrm{b}$ ). The uniformity of the size of the observed gold nanoparticles was the consequence of a homogeneous distribution of the $\mathrm{AuCl}_{4}$ - anions inside the Langmuir-Blodgett 
(LB) matrix.

Within lamellar phase which consists of stacked sheets composed of bilayers of amphiphilic molecules separated from each other by water layers, we show the possibility of producing assemblies of gold nanoparticles by gamma irradiation (Figure 7c). The particle morphology is determined by geometrical constraints, notably the thickness of water layers, and chemical constraints induced by the stabilizing amphiphilic molecules (surfactants or lipids) that compose bilayers (60).

\section{d) Templating against existing nanoparticles}

Currently existing nanoparticles with a well-controlled shape are useful as templates to generate gold replica. For instance, carbon nanotubes have been explored by Dai et al to fabricate gold nanowires (61). Yang et al have demonstrated that freestanding gold nanowires can be prepared through a redox reaction involving $\mathrm{LiMO}_{3} \mathrm{Se}_{3}$ molecular wires and $\mathrm{AuCl}_{4}$ aqueous solution (62). Using a similar approach, Xia et al have synthesized gold nanotubes (63), gold triangular nanorings (64), gold nanoboxes and gold nanocages (65), via galvanic displacement reactions between $\mathrm{HAuCl}_{4}$ and silver nanowires, triangular nanoplates and nanocubes, respectively. In this series of works, silver nanostructures of controlled morphology provide a template surface for gold nucleation and growth, imparting their shape to the resulting gold nanoparticles. Templating against existing nanoparticles provides a generic and powerful method to generate gold particles of various shapes. Nevertheless, one of the major problems associated with this approach is the difficulty in achieving a tight control over the crystallinity of the final product.

\section{e) Templating within bioreactors or against biomolecules}

It has been known for some time that plants can uptake gold from soils as they are used as bioindicators of gold deposits by mining companies (66). The study of growth of gold nanoparticles inside live plants has very important implications in ecotoxicology and possible applications, as the removal of gold (or other elements) from contaminated zones. For instance, bioreduction of gold cations inside live plants (alfalfa) leads to $4 \mathrm{~nm}$ gold icosahedrons (67). However, although plants are known to uptake gold from solution and soils, the oxidation state and the form of the gold as well as the connection between metal ions and biological systems has never been elucidated.

Shape control of gold nanoparticles using biosystems has been also investigated employing polypeptides (68), microorganisms (69) or plant extracts $(70,71,72,73)$. Specific polypeptide repeat sequences in proteins secreted by the bacterium Escherichia coli have been shown to induce growth of flat, triangular gold nanocrystals at a $4 \%$ yield relative to the total nanoparticle formation (68). A recent and unique biosynthesis method has been proposed to produce gold nanowires with a network structure using a cell-free extract of Rhodopseudomonas capsulata microorganism and to offer control over the shapes of gold nanoparticles with the change of $\mathrm{HAuCl}_{4}$ concentration (69). Inactivated alfalfa extract has also shown to be effective at passively binding and reducing gold from solutions containing $\mathrm{Au}^{3+}$ ions and resulting in the formation of $\mathrm{Au}^{0}$ nanoparticles of different shapes (Figure 8) (70).

\section{ii) Assembly of spherical nanoparticles}

Self-assembly has been extensively explored as a bottom-up approach for generating complex nanoparticles on various scales (74). In particular, spherical gold nanoparticles have been pursued as building units for the formation of structures with more complex shapes. Rudimentary gold nanowires were, for instance, obtained by sintering the nanoparticles confined within the pores of an alumina membrane at $300^{\circ} \mathrm{C}$ for several hours (75). It was demonstrated that chains of $\mathrm{Au}_{55}\left(\mathrm{PPh}_{3}\right)_{12} \mathrm{Cl}_{6}$ clusters could be formed at the waterdichloromethane interface as induced by $\operatorname{poly}(p$ phenyleneethynylene) dissolved in the liquid phase (76). If poly(vinylpyrrolidone) (PVP) was present in the solution phase, a 2-D network of cluster-loaded polymer chains was observed at the air-liquid interface under surface compression in a Langmuir-Blodgett through (77). In this case, the gold clusters were mainly trapped and organized by the intersections between entangled polymer chains.

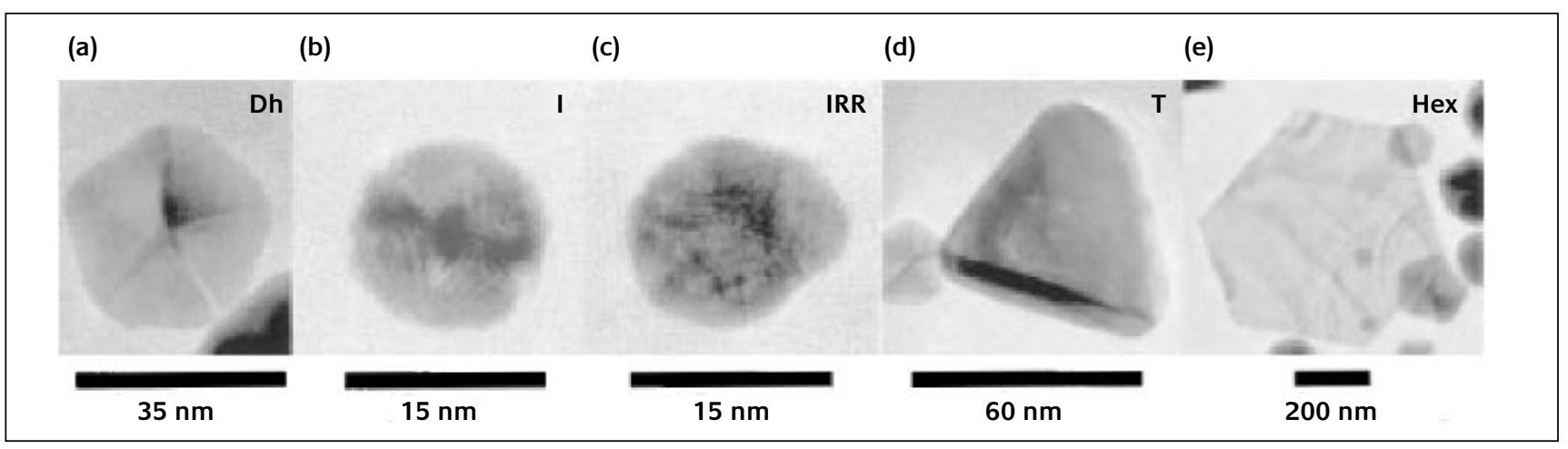

\section{Figure 8}

TEM micrographs of the different kinds of gold particles observed from the reaction of $A u^{3+}$ cations with alfalfa extracts: (a) decahedral multiple twinned; (b) icosahedral multiple twinned; (c) irregular shaped; (d) Fcc tetrahedral; (e) Fcc hexagonal platelets (70). (C) 2003 Kluwer Academic Publishers 


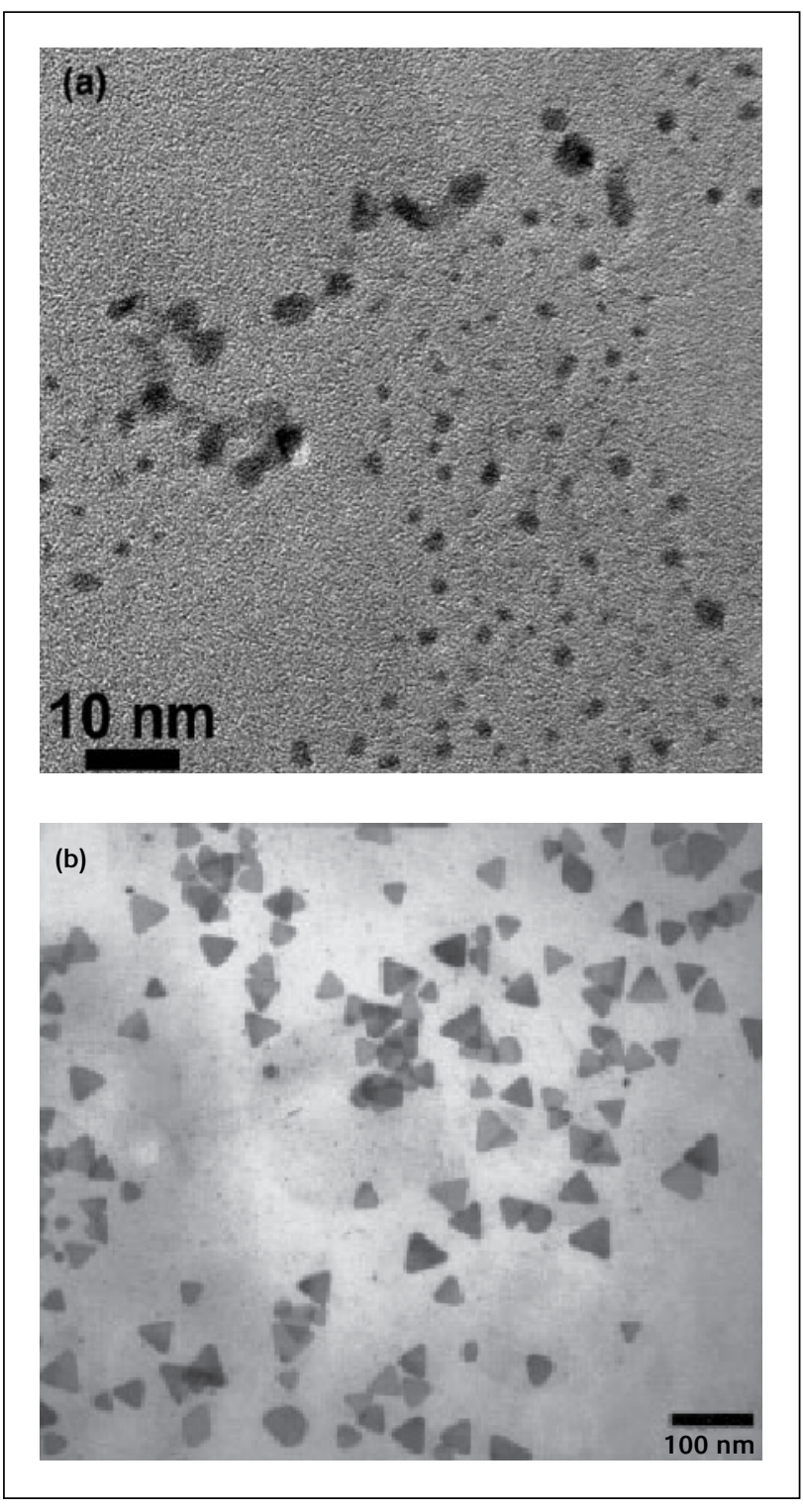

\section{Figure 9}

TEM images of gold nanoparticles grown in the presence of a) iodide ions and b) chloride ions

In addition to self-assembly, external manipulation with a field, a mechanical probe or a chemical linker has also been explored as a means of direct organization of gold nanospheres. For example, linear chains and circular rings have been fabricated by manipulating the positions of individual gold nanoparticles with the probe of a scanning force microscope (78). A very elegant approach was also developed by Feldheim et al to synthesize phenylacetylenebridged gold nanoparticle dimers and trimers in solution (79). Poly(phenylacetylene) oligomers was chosen as basic linker repeat-units because i) they are conformationally rigid molecules which could be expected to keep coupled nanoparticles at a fixed distance, ii) they can be coupled to form a variety of geometries (e.g. linear, bent, trigonal planar, tetrahedral), iii) lengths of up to 16 repeat units (ca. $20 \mathrm{~nm}$ ) are readily synthesized without significant solubility problems and iv) they have been discussed as potential wire candidates for molecular electronic devices. iii) Growth directed particle formation using capping reagent(s)

The shape of a crystal is determined by the relative specific surface energies associated with the facets of this crystal. At equilibrium, a crystal has to be bounded by facets giving a minimum total surface energy. Therefore, the shape of a single-crystalline nanostructure often reflects the intrinsic symmetry of the corresponding lattice. The shape of a crystal can also be considered in terms of growth kinetics, by which the fastest growing planes should disappear to leave behind the slowest growing ones as the facets of the product. This argument implies the final shape of a crystal can be controlled by introducing appropriate capping reagent(s) to change the free energies of the various crystallographic surfaces and thus to alter their growth rates. Xia et al have reported a polyol method to fabricate anisotropic gold nanoparticles by reducing a gold salt with ethylene glycol (EG) in the presence of PVP $(80,81,82)$. During this thermal-treatment process at higher temperature (generally outclass $100^{\circ} \mathrm{C}$ ), EG serves as solvent as well as the reducing agent of the metal precursor and PVP serves as a polymeric capping reagent to control the growing rate of different crystal facets. In succession, tetrahedral, icosahedral and cubic gold nanocrystals were also obtained by a similar PVP-EG process and by introducing foreign ions (e.g. silver ions) during the nanocrystal growth process (83). It is believed this synthetic procedure could be scaled up to produce non-spherical gold nanoparticles at the gram scale.

It is also now generally recognized that the presence of external anions, such as halogen ions, plays an important role in modulating the shape of metal nanoparticles (84). In our laboratory, we have investigated the growth of gold nanoparticles through the reduction of monovalent or trivalent gold salts by sodium sulphide in the presence of anions such as I- $\mathrm{Cl}^{-}$and $\mathrm{Br}^{-}$(85). While chloride ions promote the formation of gold nanotriangles, bromide and iodide ions distort the morphology significantly and lead to the formation of spherical particles (Figure 9). Bromide and iodide are known to be capable of replacing chemisorbed $\mathrm{Cl}^{-}$on gold surface and thus potentially control the rate of growth of the nuclei. Our comparison of the shape distributions obtained in the different experimental conditions lead us to the conclusion that the control of the shape of gold nuclei is more important than the control of the growth rate of a specific plane in obtaining gold nanoparticles of the desired shape.

We have also investigated the formation of triangular gold particles under the electron beam by in-situ high-resolution TEM (86). Triangular gold formation was observed through the melting of two gold nanoparticles onto copper grid support. The melting temperature of nanometer-sized gold particles is lowered by several hundreds of degrees (87). Particle of about $2 \mathrm{~nm}$ in diameter have a melting temperature below $573 \mathrm{~K}$, which could typically be reached under ultra vacuum conditions and with a sufficient focused TEM electron beam. At the beginning of the process, the smaller twinned 
gold particle rotates slightly to reduce the gap between the two particles (Figure 10). Within a few seconds, two parallel interface planes are created and a weak bridge is formed, rapidly followed by a neck. The structure and the orientation of the particle change and the latter is "swallowed" by the gold tabular particle. At the end of the process, the upper right angle is formed and the triangular form of the gold tabular particle is now better defined.

Investigations of the optical properties of those triangular particles have shown that the surface plasmon bands frequency is strongly sensitive to the corner sharpness (Figure 11). Simulations by Discrete Dipolar Approximation (DDA) approach qualitatively indicate that the electric field is mainly associated with the particle corners. The formation of truncated corners leads to a more diffuse electromagnetic field and thus a strong change of optical properties. Similar effects were also emphasized for silver nanoparticles (88).

Over their ability to form micelles (i.e. soft template), surfactant molecules, or complex ion versions of them, can also potentially bind to specific crystal faces and direct nanocrystal growth (89-90). Murphy et al have demonstrated that at some stage of growth of gold nanorods, the competitive and preferential binding of CTAB molecules on a certain face of gold may lead to the generation of faceted spheres, which evolve to form 1D nanostructures (91). Different surfactants can be used either singly or in combination with others to vary the shape and size of the produced nanorods (92). Different factors such as the head group of the surfactant and chain length all affect the nature of the nanorods produced, making predictions about surfactant choice difficult. Alkyltrimethylammonium bromides are effective surfactants that assist in the synthesis of gold nanorods of various sizes $(56,93,94)$. CTAB appears to be a popular choice. After synthesis, the gold nanorods are capped with a bilayer of CTAB in which the polar head of one monolayer faces the gold, the other polar head faces the aqueous solvent, and the non-polar tails interact to sequester themselves from water (95). Hence, the formation and stability of the nanorods depend on the head group of the surfactant (which binds to the nanorods) and also on the tail of the surfactant (hydrophobic/van der Waals interaction between hydrocarbon chains). Investigation of the variation of hydrocarbon tail length in tetraalkylammonium surfactants ( $\left.C_{n} T A B\right)$ shows a surprisingly strong dependence of the gold nanorod aspect ratio on tail length (96). This indicates the role of van der Waals stabilization of the surfactant bilayer on the gold surface due to interchain packing, which assists in the nanorod formation.

\subsection{Top-down routes}

Natural lithography, renamed nanosphere lithography (NSL) (97) and colloidal lithography (98), is a powerful technique to inexpensively produce nanoparticle arrays with controlled shape, size and interparticle spacing. NSL is based on the selfassembly of size-monodispersed nanospheres, often polystyrene latexes, to form a two dimensional colloidal crystal deposition mask on a Si substrates. As the solvent evaporates, capillary forces draw the nanospheres together, and the nanosphere crystallise into a hexagonally closepacked pattern on the substrate. Following self-assembly of the nanosphere mask, gold is deposited by thermal evaporation, electron beam deposition or pulsed laser deposition. The mask is then removed, leaving surfaceconfined nanoparticles with triangular footprints. Specifically, hexagonal arrays of nanotriangles are formed from a singlelayered colloidal mask, and spherical dot arrays with different unit lattices are fabricated from a double-layered mask $(99,100)$. In this case, the substrate to be patterned is

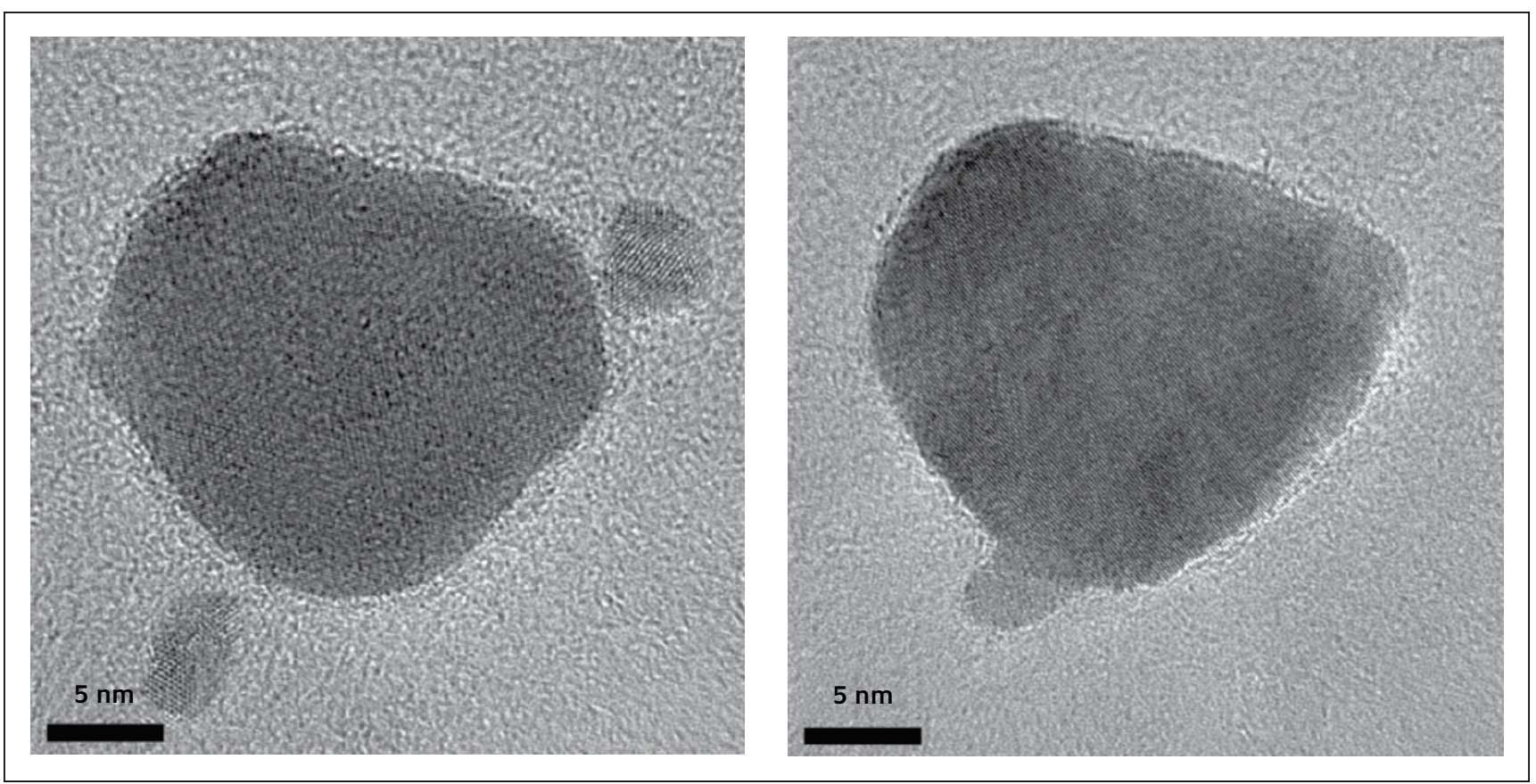

\section{Figure 10}

HRTEM micrographs extracted from the video sequence of gold nanoparticles sintering before and after intense electron beam exposure (86) 
positioned normal to the direction of material deposition. By controlling the angle between the surface normal of the sample assembly and the propagation vector of the material deposition beam, vastly different and increasingly flexible nanostructures such as chains, gap, contact and overlap nanostructures have been generated (99). Cold nanoring arrays were also found on the silicon wafer substrate by using the random incidence sputtering deposition technique on patterned polystyrene microspheres (101).

NSL is often combined with another technique to create new patterned nanomasks. By means of reactive ion etching, the dimension of the nanosphere can be reduced without changing the center-to-center distance of the nanospheres $(100,102)$. Thermal annealing can also be used to transform the shape of the resulting nanoparticles from triangular gold plates to rounded gold nanoparticles (100). Cold nanodisks arranged in planar arrays have been prepared using colloids directly as masks (102). This technique called hole mask nanolithography consists in several steps: (i) the deposition of polystyrene particles by electrostatic self-assembly onto glass slides precoated with $20 \mathrm{~nm}$ of $\mathrm{Au}$. The resulting particle saturation coatings have well-defined structure with short range ordering due to electrostatic interparticle repulsion. At this step, the interparticle separation can be controlled by the salt concentration in the colloidal solution. (ii) The shape and size of the adsorbed polystyrene particles are modified by heat treatment, a few minutes on a hotplate, or by oxygen plasma treatment. (iii) Finally, $\mathrm{Ar}$ ion beam etching is used to remove the Au film, utilizing the particle film as an etch mask. The remains of the polystyrene particles are removed by a UV/ozone treatment and a subsequent water rinse, leaving separated oblate gold disks on the glass. Hybrid nanostructures (disk shape) composed of metal dielectric nano-sandwiches (gold-silica) have been produced with this technique for the study of bimodal resonant response at optical frequencies observed due to dipolar plasmon hybridization (103).

Dip-pen nanolithography was also used to generate layers on $\mathrm{Au}$, combined with wet-chemical etching to fabricate nanostructures with nanoscale precision and deliberately designed shapes (lines, dots, rings, and triangles), sizes and

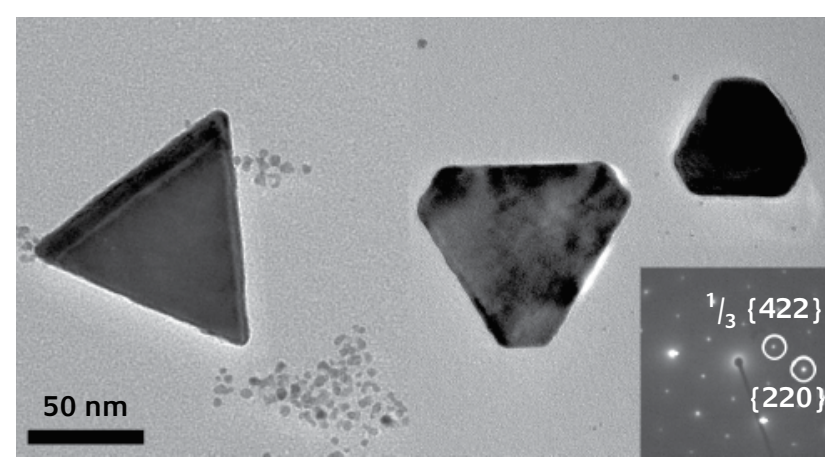

\section{Figure 11}

TEM image of gold triangular particles with different corner sharpness (inset: diffraction pattern). (85) nanoscale precision $(104,105)$. Other lithographic methods have been described in the literature combining several techniques. As an example, a very recent and original method termed on-wire lithography, combining advances in templatedirected synthesis of nanowires with electrochemical deposition and wet-chemical etching allows routine fabrication of face-to-face disk arrays and gap structures in the range of five to several hundred nanometers $(106,107)$. Physical methods can be also cited here, combining ion-beam and electron beam, used for preparing gold nanostructures difficult to prepare by classical wet chemistry routes such as gold micro-hole arrays (108), grooves and nanowires circuits (109) in order to study their plasmon modes, the propagation of surface plasmons $(108,109,110)$ and to demonstrate experimentally the existence of negative refractive index for optical range for metamaterials based on nanostructured gold nanowires $(111,112,113)$.

\section{Key challenges}

Chemical synthesis of gold nanoparticles with well-controlled shapes, sizes and structures is becoming a practical reality. The development of a colloid chemistry route continues to be essential for the synthesis and manipulation of anisotropic gold nanoparticles, with major requirements already demonstrated, such as the control of the nuclei shape, the growth on specific facets, etc. Further efforts should focus on a better understanding of the growth mechanisms for best shape selectivity. Many mechanistic issues of gold growth need to be addressed such as crystal structure effects of the seeds on the resulting nanoparticles, twinning, the role of surfactant (template role, inhibitor role or electric field role ?), etc.

The gold nanoparticles described in this paper likely represent just a few paths toward full tunability. An interesting aspect which has not yet been fully explored is the synthesis of Janus-like gold nanoparticles, that is, nanoparticles bearing different surface functions on each of their hemispheres (114). Original assembling ability and interesting optical properties are expected as some recent papers seem to demonstrate $(115,116,117,118)$.

The study of a sample with narrow size and shape distribution is essential for determining the physical and chemical properties of individual particles in assemblies and mixtures. A great effort is needed to create assemblies of nanoparticles in two or three dimensional structures. Observations of new optical properties of self-assembled monolayers of anisotropic gold structures are expected as now the electric fields connected to the plasmon resonance will influence the effective fields around the neighbouring nanoparticles. With continued development in their synthesis and assembly, gold nanoparticles will be a standard stock of future photonics, biomedical and catalytic applications.

Lastly, the next challenge will be the integration of such anisotropic gold nanoparticles into devices that would take advantage of their unique properties. 


\section{About the authors}

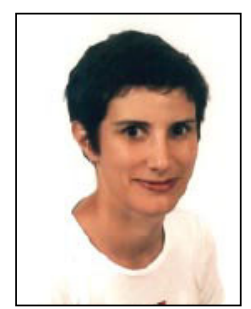

Mona Tréguer-Delapierre is assistant Professor of inorganic chemistry at the University of Bordeaux I and developed her research at the Institute of Condensed Matter Chemistry of Bordeaux (France). Her research interests include the synthesis of metal based nanomaterials with interesting optical properties for optics and biomedical applications.

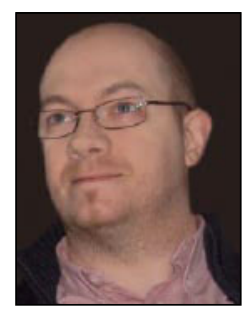

Dr Jérôme Majimel is currently CNRS researcher at the Institute of Condensed Matter Chemistry in Bordeaux. His research activities are focused on the characterization of metal-based nanocomposite systems, with a particular attention being paid to the surfaces and interfaces mainly using Transmission Electron Microscopy in its different modes: conventional, high resolution, in-situ and analytic.

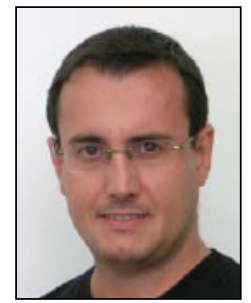

Stéphane Mornet is currently researcher at the French National Center for Scientific Research (CNRS) at the Institute of Condensed Matter Chemistry of Bordeaux and his research, at the interface of chemistry and biology, focuses on the synthesis of magnetic, metallic and luminescent nanoparticles, their surface functionalization and conjugation with biomolecules for imaging and therapy purposes.

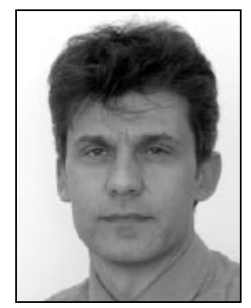

Prof. Etienne Duguet is employed at the University of Science and Technology of Bordeaux. His research focuses on the synthesis and characterization of hybrid organic-inorganic materials based on inorganic particles derivatized through molecular surface modification, polymer encapsulation, dissymetrization and/or functionalisation for optical or medical applications.

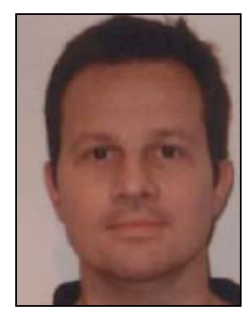

Serge Ravaine received an engineer diploma from the National School of Chemistry and Physics of Bordeaux in 1991 and a PhD in Physical Chemistry at the University of Bordeaux in 1995. He is currently full professor at the University of Bordeaux and his research interests at the Centre de Recherche Paul Pascal in Pessac (France) include the synthesis of hybrid colloidal particles and the fabrication of three-dimensional colloidal photonic crystals.

\section{References}

1 C. Murphy, A.M. Gole, S. Hunyadi, C. Orendorff, Inorg. Chem, 2006, 45 7544

2 C. Burda, X. Chen, R. Narayan, M.A. El-Sayed, Chem. Rev. 2005, 105, 1025

3 Y. Sun, Y. Xia, Science, 2002, 298, 2176

4 S.E. Skrabalak, J. Chen, L. Au, X. Lu, X. Li, Y. Xia, Adv. Mater., 2007, 19, 3177

5 S.Oldenburg, R.D. Averitt, S.L. Westcott, N.J. Halas, Chem. Phys. Lett., 1998, 288, 243

6 S. Shankar, S. Bhargav, M. Sastry, J. Nanosci. Nanotech., 2005, 5, 1721

7 N. Malikova, I.Pastoriza-Santos, M. Schierhorn, N. Kotov, L. Liz-Marzan, Langmuir, 2002, 18, 3694

8 L. Manna, D. Milliron, A. Meisel, E.C. Scher, A.P. Alivisatos, Nature Mater., $2003,2,382$

9 E. Hao, R.C. Balley, G.C. Schatz, J. Hupp, S. Li, NanoLett., 2004, 4, 327

10 M. Hu, J. Chen, Z. Li, L. Au, G.V. Hartland, X. Li, M. Marquez, Y. Xia, Chem. Soc. Rev., 2006, 35, 1084

11 S.J. Son, X. Bai, S.B. Lee, Drug Delivery Today, 2007, 12, 657

12 G.C. Bond, C. Louis, D.T. Thompson, Catalysis by Gold, London Imperial College Press, 2006, Catalytic Science Series, Vol 6, Imperial College Press, p. 366

13 L. Liz-Marzan, Langmuir, 2006, 22, 32

14 C.J. Murphy, T.K. Sau, A.M. Gole, C.J. Odendorff, J. Gao, L. Gou, S.E. Hunyadi, T. Li, J. Phys. Chem. B, 2005, 109, 13857

15 J. Pérez-Juste, I. Pastariza-Santos, L. Liz-Marzan, P. Mulvaney, Coord. Chem. Rev., 2005, 249, 1807

16 A. Wei, e-J. Surf. Sci. Nanotech., 2006, 4, 9

17 I. Willner, e-J. Surf. Sci. Nanotech., 2005, 3, 1

18 S. Link, M. El Sayed, Int. Rev. Phys. Chem., 2000, 19, 409

19 S. Lee, Y. Yun, S. Cho, J. Cheon, J. Am. Chem. Soc., 2002, 124, 11244

20 J. Yang, J.H. Fendler, J. Phys. Chem., 1995, 99, 5505

21 S.O. Obare, N.R. Jana, C.J. Murphy, Nano Lett., 2001, 1, 601

22 G. Metraux, Y. Cao, R. Jin, C. Mirkin, Nano Lett., 2003, 3, 519

23 J. Pérez-Juste, P. Mulvaney, Int. J. Nanotechnol., 2007, 4, 215

24 EOS Topical Meeting on Molecular Plasmonic Devices, April 27-29, 2006, Engelberg, Switzerland (European Optical Society, 2006)

25 K. Imura, T. Nagahara, H. Okamoto, J. Am. Chem. Soc., 2004, 126, 12730

26 K.L. Kelly, E. Coronado, L.L Zhao, G.C. Schatz, J. Phys. Chem. B, 2003, 107, 668

27 P. Hanarp, M. Kall, D.S. Sutherland, J. Phys. Chem. B, 2003, 107, 5768

28 M.S. Chen, D.W. Goodman, Catal. Today, 2006, 111, 22

29 R. Narayanan, M.A. El-Sayed, Nano Lett., 2004, 7, 1343

30 H. Rashid, R.R. Bhattachargee, A. Kotal, T. Mandal, Langmuir, 2006, 22, 7141

31 M.C. Daniel, D. Astruc, Chem. Rev., 2004, 104, 293

32 J. Turkevitch, P. Stevenson, J. Hillier, Discuss. Faraday Soc., 1951, 11, 55

33 J. Henzie, E.S. Kwak, T.W. Odom, Nano Lett., 2005, 5, 1199

34 R.M. Penner, J. Phys. Chem. B, 2002, 106, 3339

35 E.C. Walter, B.J. Murray, F. Favier, G. Kaltenpoth, M. Grunze, R.M. Penner, J. Phys. Chem. B., 2002, 106, 11407

36 J. Majimel, D. Bacinello, E. Durand, F. Vallée, M. Tréguer-Delapierre, Langmuir, 2008, 24, 4289

37 J. Majimel, M. Lamirand-Majimel, I. Moog, C. Feral-Martin, M. TréguerDelapierre, submitted

38 S. Bernal, FJ. Botana, J.J. Calvino, G.A. Cifredo, J.A. Pérez-Omil, J.M. Pintado, Catal. Today, 1995, 23, 219 
C.A. Foss Jr., G.L. Hornyak, J.A. Stockert, C.R. Martin, Adv. Mater., 1993, 5, 135.

44 R.M. Penner, C.R. Martin, Anal. Chem., 1987, 59, 2625

45 B.M.I. van der Zande, M.R. Boehmer, L.G.J. Fokkink, C. Schoenenberger, J. Phys. Chem. B, 1997, 101, 852

46 V.M. Cepak, C.R. Martin, J. Phys. Chem. B, 1998, 102, 9985

47 K.B. Jirage, J.C. Hulteen, C.R. Martin, Science, 1997, 278, 655

48 B.M.I. van der Zande, M.R. Boehmer, L.G.J. Fokkink, C. Schoenenberger, Langmuir, 2000, 16, 451

49 C. Schoenenberger, B.M.I. van der Zande, L.G.J. Fokkink, M. Henny, C. Schmid, M. Krueger, A. Bachtold, R. Huber, H. Birk, U. Staufer, J. Phys. Chem. B, 1997, 101, 5497

50 Z. Hou, N.L. Abbott, P. Stroeve, Langmuir, 2000, 16, 2401

51 M. Wirtz, C.R. Martin, Adv. Mater., 2003, 15, 455

52 F. Yan, W.A. Goedel, Nano Lett., 2004, 4, 1193

53 G. Lu, R. Zhao, G. Qian, Y. Qi, X. Wang, J. Suo, Catal. Lett., 2004, 97, 115

54 Y.Y. Yu, S.S. Chang, C.L.Lee, C.R.C. Wang, J. Phys. Chem. B, 1997, 101, 6661

55 M.A. El-Sayed, Acc. Chem. Res., 2001, 34, 257

56 N.R. Jana, L. Gearheart, C.J. Murphy, J. Phys. Chem. B, 2001, 105, 4065

57 B.D. Busbee, S.O. Obare, C.J. Murphy, Adv. Mater., 2003, 15, 414

58 M. Antonietti, A. Thünemann, E. Wenz, Coll. Polym. Sci., 1996, 274, 795

59 S. Ravaine, G.E. Fanucci, C.T. Seip, J.H. Adair, D.R. Talham, Langmuir, 1998, 14, 708

60 M.E. Meyre, M. Tréguer-Delapierre, C. Faure, Langmuir, 2008, 24, 4289

61 Y. Zhang, H. Dai, Appl. Phys. Lett., 2000, 77, 3015

62 J.H. Song, Y. Wu, B. Messer, H. Kind, P. Yang, J. Am. Chem. Soc., 2001, 123, 10397

63 Y. Sun, B.T. Mayers, Y. Xia, Nano Lett., 2002, 2, 481

64 Y. Sun, Y. Xia, Adv. Mater., 2003, 15, 695

65 S.E. Skrabalak, J. Chen, L. Au, X. Lu, X. Li, Y. Xia, Adv. Mater., 2007, 19, 3177

66 B.I.A. Mclnnes, C.E. Dunn, E.M. Cameron, L. Kameko, J. Geochem. Explor., 1996, 57, 227

67 J.L. Gardea-Torresdey, J.G. Parsons, E. Gomez, J. Peralta-Videa, H.E. Troiani, P. Santiago, M. José Yacamán, Nano Lett., 2002, 2, 397

68 S. Brown, M. Sarikaya, E. Johnson, J. Mol. Biol., 2000, 299, 725

69 S. He, Y. Zhang, Z. Guo, N. Gu, Biotechnol. Prog., in press (DOI: 10.1021/ bp0703174)

70 J.L. Gardea-Torresdey, K.J. Tiemann, G. Gamez, K. Dokken, S. Tehuacanero, M. José Yacamán, J. Nanopart. Res., 1999, 1, 397

71 S.S. Shankar, A. Rai, B. Ankamwar, A. Singh, A. Ahmad, M. Sastry, Nature Mater., 2004, 3, 482

72 S.S. Shankar, A. Rai, A. Ahmad, M. Sastry, Chem. Mater., 2005, 17, 566

73 S.P. Chandran, M. Chaudhary, R. Pasricha, A. Ahmad, M. Sastry, Biotechnol. Prog., 2006, 22, 577

74 L. Isaacs, D.N. Chin, N. Bowden, Y. Xia, G.M. Whitesides, in Supermolecular Technology (Ed: D.N. Reinhoudt), John Wiley\&Sons, New York, 1999, pp. 1-46

75 G. Hornyak, M. Kroll, R. Pugin, T. Sawitowski, G. Schmid, J.O. Bovin, G. Karsson, H. Hofmeister, S. Hopfe, Chem. Eur. J., 1997, 3, 1951
76 D. Wyrwa, N. Beyer, G. Schmid, Nano Lett., 2002, 2, 419

77 T. Reuter, O. Vidoni, V. Torma, G. Schmid, L. Nan, M. Gleiche, L. Chi, H. Fuchs, Nano Lett., 2002, 2, 709

78 R. Resch, C. Baur, A. Bogacov, B.E. Keol, P.M. Echternach, A. Madhukar, N. Montoya, A.A.G. Requicha, P. Will, J. Phys. Chem. B, 1999, 103, 3647

79 L.C. Brousseau, J.P. Novak, S.M. Marinakos, D.L. Feldheim, Adv. Mater., 1999, 11, 447

80 Y. Sun, Y. Xia, Science, 2002, 298, 2176

81 Y. Sun, B. Gates, Y. Xia, Nano Lett., 2002, 2, 165

82 Y. Sun, Y. Xia, Adv. Mater., 2002, 14, 833

83 F. Kim, S. Connor, H. Song, T. Kuykendall, P. Yang, Angew. Chem. Int. Ed., 2004, 43, 3673

84 S.S. Shankar, S. Bhargava, M. Sastry, J. Nanosci. Nanotech., 2005, 5, 1721

85 C. Feral-Martin, J. Majimel, M. Tréguer-Delapierre, F. Vallée, G. Bachelier, unpublished results

86 J. Majimel, C. Feral-Martin, M. Tréguer-Delapierre, video accessible at http://tem.eklablog.com/article-29012-frittage-de-nanoparticules-sousfaisceau.html

87 P. Buffat, J.P. Borel, Phys. Rev. A, 1976, 13, 2287

88 K.L. Kelly, E. Coronado, L.L Zhao, G.C. Schatz, J. Phys. Chem. B, 2003, 107, 668

89 C.J. Johnson, E. Dujardin, S.A. Davis, C.J. Murphy, S. Mann, J. Mater. Chem., 2002, 12, 1765

90 P.L. Gai, M.A. Harmer, Nano Lett., 2002, 2, 771

91 C.J. Murphy, T.K. Sau, A. Gole, C.J. Orendorff, MRS Bull., 2005, 30, 349

92 B. Nikoobakht, M.A. El-Sayed, Chem. Mater., 2003, 15, 1957

93 N.R. Jana, L. Gearheart, C.J. Murphy, Chem. Commun., 2001, 7, 617

94 N.R. Jana, L. Gearheart, C.J. Murphy, Adv. Mater., 2001, 13, 1389

95 B. Nikoobakht, M.A. El-Sayed, Langmuir, 17, 6368

96 J. Gao, C.M. Bender, C.J. Murphy, Langmuir, 2003, 19, 9065

97 C.L. Haynes, R.P. van Duyne, J. Phys. Chem. B, 2001, 105, 5599

98 S.M. Yang, S.G. Jang, D.G. Choi, S. Kin, H.K. Yu, Small, 2006, 2, 458

99 C.L. Haynes, R.P. van Duyne, Nano Lett., 2003, 3, 939

100 B.J.Y. Tan, C.H. Sow, T.S. Koh, K.C. Chin, A.T.S. Wee, C.K. Ong, J. Phys. Chem. B, 2005, 109, 11100

101 D. Jia, A. Goonewardene, Appl. Phys. Lett., 2006, 88, 053105

102 P. Hanarp, M. Käll, D.S. Sutherland, J. Phys. Chem. B, 2003, 107, 5768

103 A. Dmitriev, T. Pakizeh, M. Käll, D.S. Sutherland, Small, 2007, 3, 294

104 H. Zhang, K.B. Lee, Z. Li, C.A. Mirkin, Nanotech., 2003, 14, 1113

105 H. Zhang, C.A. Mirkin, Chem. Mater., 2004, 16, 1480

106 L. Qin, S. Park, L. Huang, C.A. Mirkin, Science, 2005, 309, 113

107 L. Qin, S. Zou, C. Xue, A. Atkinson, G.C. Schatz, C.A. Mirkin, PNAS, 2006, 103, 13300

108 E. Devaux, T.W. Ebbesen, J.C. Weeber, A. Dereux, Appl. Phys. Lett., 2003, 83, 4936

109 W.L. Barnes, A. Dereux, T.W. Ebbesen, Nature, 2003, 424, 824

110 S.I. Bozhevolnyi, V.S. Volkov, E. Devaux, J.Y. Laluet, T.W. Ebbesen, Nature, 2006, 440, 508

111 V.M. Shalaev, W. Cai, U.K. Chettiar, H.-K. Yuan, A.K. Sarychev, V. Drachev, A.V. Kildishev, Opt. Lett., 2005, 30, 3356

112 G. Dolling, C. Enkrich, M. Wegener, J.F. Zhou, C.M. Soukoulis, S. Linden, Opt. Lett., 2005, 30, 3198

113 V.A. Podolskiy, A.K. Sarychev, V.M. Shalaev, J. Nonlinear Opt. Phys. Mater., 2002, 11, 65

114 A. Perro, S. Reculusa, S. Ravaine, E. Bourgeat-Lami, E. Duguet, J. Mater. Chem., 2005, 15, 3745 
115 J. van Herrikhuyzen, G. Portale, J.C. Gielen, P.C.M. Christianen, N.A.J.M. Sommerdijk, S.C.J. Meskers, A.P.H.J. Schenning, Chem. Comm., 2008, 2008, 697

116 S. Pradhan, L.P. Xu, S.W. Chen, Adv. Funct. Mater., 2007, 17, 2385

117 C. Vilain, F. Goettmann, A. Moores, P. Le Floch, C. Sanchez, J. Mater. Chem., 2007, 17, 3509

118 D. Suzuki, H. Kawaguchi, Coll. Polym. Sci., 2006, 284, 1471 